\title{
Melatonin Modulation of Sirtuin-1 Attenuates Liver Injury in a Hypercholesterolemic Mouse Model
}

\author{
Francesca Bonomini, ${ }^{1,2}$ Gaia Favero ${ }^{(D)},{ }^{1}$ Luigi Fabrizio Rodella $\left(\mathbb{D},{ }^{1,2}\right.$ \\ Mohammed H. Moghadasian, ${ }^{3}$ and Rita Rezzani $\complement^{1,2}$ \\ ${ }^{1}$ Anatomy and Physiopathology Division, Department of Clinical and Experimental Sciences, University of Brescia, Viale Europa 11, \\ 25123 Brescia, Italy \\ ${ }^{2}$ Interdipartimental University Center of Research “Adaption and Regeneration of Tissues and Organs (ARTO)", University of Brescia, \\ Viale Europa 11, 25123 Brescia, Italy \\ ${ }^{3}$ Department of Human Nutritional Sciences, University of Manitoba and Canadian Centre for Agri-Food Research in \\ Health and Medicine, St. Boniface Hospital Research Centre, Winnipeg, MB, Canada \\ Correspondence should be addressed to Rita Rezzani; rita.rezzani@unibs.it
}

Received 26 September 2017; Accepted 4 January 2018; Published 4 February 2018

Academic Editor: Antoni Camins

Copyright (C) 2018 Francesca Bonomini et al. This is an open access article distributed under the Creative Commons Attribution License, which permits unrestricted use, distribution, and reproduction in any medium, provided the original work is properly cited.

Hypercholesterolemia increases and exacerbates stress signals leading also to liver damage (LD) and failure. Sirtuin1 (SIRT1) is involved in lifespan extension and it plays an essential role in hepatic lipid metabolism. However, its involvement in liver hypercholesterolemic damage is not yet completely defined. This in vivo study evaluated the role of SIRT1 in the hypercholesterolemic-related LD and, then, investigated how oral supplementation of melatonin, pleiotropic indoleamine, may be protective. Control mice and apolipoprotein E-deficient mice $\left(\mathrm{ApoE}^{-/-}\right)$of 6 and 15 weeks of age were treated or not treated with melatonin at the dose of $10 \mathrm{mg} / \mathrm{kg} /$ day for 9 weeks. In this study, we evaluated serum biochemical markers, liver SIRT1 expression, and oxidative stress markers. We observed that hypercholesterolemia increased significantly serum cholesterol and triglycerides, reduced significantly liver SIRT1, and, in turn, induced hepatic oxidative stress in untreated ApoE ${ }^{-/-}$mice with respect to control mice. Interestingly, melatonin treatment improved serum biochemical markers and hepatic morphological impairment and inhibited oxidative stress through its antioxidant properties and also by SIRT1 upregulation. In summary, melatonin oral supplementation may represent a new protective approach to block hypercholesterolemic liver alterations involving also a SIRT1dependent mechanism.

\section{Introduction}

Hyperlipidemia, which included hypercholesterolemia (HC), is the most striking risk factor in the development of cardiovascular diseases in occidental population [1]. Besides its correlation with cardiovascular events, HC is considered a risk factor that contributes to liver damage (LD). Moreover, this disorder has been reported in $20 \%-80 \%$ of cases of nonalcoholic fatty liver disease (NAFLD) [2] and may induce liver inflammatory cell infiltration, fibrosis, and production of chemokines, cytokines, and oxidative factors that, in turn, lead to oxidative stress (OS) and inflammation [3-5]. OS, mitochondrial dysfunction, and upregulation of proinflammatory cytokines have been suggested to be the major consequences of cellular lipid overload $[6,7]$, contributing, in turn, to hepatic inflammatory damage and fibrogenesis $[8,9]$. Moreover, OS characterized by decreased activity of endogenous antioxidant system, with a reduction of superoxide dismutasel (SOD1) and catalase (CAT), together with an increase in inducible nitric oxide synthase (iNOS), appears to be responsible for lipid-related LD in different experimental models [10-12]. This is consistent with previous findings from our and other research groups showing that hypercholesterolemic mice have important morphological changes at liver level, like disorganization of hepatic parenchyma with widespread cell swelling, congestion of 
sinusoids, hepatocytes fatty deposits, polyploidy, and augmented nuclear size $[13,14]$.

Recently, it has been reported that sirtuins, belonging to silent information regulator 2 family, play a key role in the development and rescue of various metabolic diseases, including NAFLD [15-17]. In fact, sirtuin1 (SIRT1) is involved in the prevention of the development of NAFLD through its role in the regulation of inflammation and lipid metabolism. SIRT1 regulates lipid metabolism through both its deacetylase activity and its direct and indirect involvement in insulin signaling [18]. Furthermore, SIRT1 modulates activities of many hepatic gene-regulatory proteins and it may activate gluconeogenesis and fatty acid oxidation or induce the lipogenesis inhibition in experimental conditions [19, 20]. Liverspecific deletion of SIRT1, as well as protein downregulation, resulted in hepatic steatosis, inflammation, and OS [21, 22].

It is known that the inhibition of SIRT1 signaling in human fetal hepatocytes induces an increase in intracellular glucose and lipid levels with upregulation of de novo lipogenesis and gluconeogenesis related genes [23]. Moreover, the hepatic deletion of SIRT1, as well as SIRT1 downregulation, caused hepatic steatosis and inflammation [24].

Currently available therapies for controlling hyperlipidemia, such as fibrates, bile acid sequestraints, and statins, are almost inefficient in lipid metabolism regulation and cause different side effects in patients [25]. Worldwide efforts aimed at reducing the impact of hyperlipidemia are among the top priorities and, to date, several strategies including nutritional supplements and anti-inflammatory treatments have been suggested to counteract HC [26].

Melatonin is an indoleamine produced and secreted into blood stream during dark period by the pineal gland. Its physiological effects include regulation of seasonal reproduction, body weight, and energy balance [27, 28]. Furthermore, melatonin acts as a highly effective antioxidant and anti-inflammatory molecule [29] with beneficial actions also against obesity and related $\operatorname{LD}[30,31]$. Further, it is known that melatonin increases, in different experimental conditions, the hepatic level of SIRT1 as well as in vessels of apolipoprotein E-deficient mice $\left(\mathrm{ApoE}^{-/-}\right)[32,33]$.

Since the mechanisms involved in $\mathrm{HC}$-related LD are not fully understood, the aim of this study was to better investigate liver morphological alterations during HC. Then, we evaluated if melatonin is effective in reducing LD and OS through also the induction of SIRT1 expression. To address this issue, we used $\mathrm{ApoE}^{-/-}$mice, an animal model that spontaneously develops $\mathrm{HC}$, aortic lipid accumulation, and LD in a time-dependent manner. As previously shown, $\mathrm{ApoE}^{-/-}$mice develop HC from the 4th week of life and then showed alterations in hepatic morphology and protein expressions [34-37].

In the present study, we provided evidence that melatonin restores liver cytoarchitecture preventing SIRT1 reduction. Furthermore, SIRT1 is able to decrease OS inducing also antioxidants expression. Then, we suggested that melatonin may be a valuable protective alternative strategy for minimizing the $\mathrm{LD}$ related to $\mathrm{HC}$, via also a SIRT1-dependent mechanism of action.

\section{Materials and Methods}

2.1. Animal Treatment. Forty C57BL/6 male mice and thirty $\mathrm{ApoE}^{-/-}$male mice (Harlan Laboratories S.r.l., Udine, Italy and Charles River Laboratories S.r.l., Lecco, Italy) were housed in an animal experimental unit with $12 \mathrm{~h}$ alternating light-dark cycle and constant temperature. Animal had free access to food and water. All the protocols were approved by the Italian Ministry of Health and complied with "Guiding Principles in the Use of Animals in Toxicology," which were adopted by the Society of Toxicology in 1989.

Mice were randomly divided into the following seven groups (ten animals per group): (1) control untreated C57BL/ 6 mice which were 6 weeks old at sacrifice; (2) control untreated C57BL/ 6 mice which were 15 weeks old at sacrifice; (3) control C57BL/6 mice treated orally with $1 \%$ ethanol (melatonin vehicle) dissolved in tap water from the 6th to 15 th week of life; (4) control C57BL/6 mice treated orally with melatonin (10 mg/kg/day) from the 6 th to 15 th week of life; (5) $\mathrm{ApoE}^{-/-}$untreated mice which were 6 weeks old at sacrifice $\left(\mathrm{ApoE}^{-/-}\right.$of $\left.6 \mathrm{w}\right)$; (6) $\mathrm{ApoE}^{-/-}$untreated mice which were 15 weeks old at sacrifice (ApoE ${ }^{-/-}$of $15 \mathrm{w}$ ); and (7) $\mathrm{ApoE}^{-/-}$mice treated orally with melatonin $(10 \mathrm{mg} / \mathrm{kg} / \mathrm{day})$ from the 6 th to 15th week of life (ApoE $\left.{ }^{-/-}+\mathrm{MEL}\right)$.

Animals were individually housed in cages with a single water bottle to ensure that all received the correct melatonin dose according to the body weight of the animal. The bottles were wrapped in aluminium foil to protect melatonin from light. Synthetic melatonin was dissolved in $1 \%$ ethanol and then diluted in tap water to yield a final dose of $10 \mathrm{mg} / \mathrm{kg}$ body weight/day, as previously described by Rezzani et al. [38].

At the end of the study, all the animals were euthanized and blood and liver samples were collected. Serum cholesterol and triglyceride concentrations were determined by standard laboratory procedures and also serum SOD1 and glutathione (GSH) levels were evaluated, as described later. Furthermore, liver samples were weighted and then fixed in $4 \%$ paraformaldehyde and embedded in paraffin wax, according to standard procedures [38, 39]. Serial sections were cut using a microtome ( $7 \mu \mathrm{m}$ thickness) for the following described morphological, immunohistochemical/immunofluorescence, and histomorphometrical analyses.

2.2. Haematoxylin-Eosin Staining. Liver paraffin-embedded sections were deparaffinized, rehydrated, and then stained with haematoxylin-eosin, following standard protocols. In detail, haematoxylin-eosin staining was used to evaluate liver morphology and also to measure the hepatocyte nuclear area $[13,40]$. The hepatocyte nuclear area was calculated using a computerized image analyzer (Image Pro Premier 9.1, Media Cybernetics Inc., Rockville, USA) evaluating 20 randomly chosen liver fields per experimental animal. Two blinded investigators performed the morphometrical analysis and their evaluation was assumed to be correct if the values were not significantly different. If there was disagreement concerning the interpretation, the case was reconsidered to reach a unanimous agreement. 
2.3. Picrosirius Red Staining. Picrosirius red staining was used to evaluate liver fibrosis [41]. In detail, paraffinembedded liver sections, after deparaffinization and rehydration, were stained for 5 minutes in 1\% phosphomolybdic acid aqueous solution and then for 3 minutes in 6\% Sirius red aqueous solution. Polarized light allowed visualization of collagen fibers of different thickness with different colours, as previously described [34, 42].

2.4. Immunohistochemistry and Double Immunofluorescence Analyses. Liver paraffin-embedded sections were deparaffinized, rehydrated, and incubated for 1 hour at room temperature in specific normal serum. Subsequently, the sections were incubated overnight at $4^{\circ} \mathrm{C}$ with the following primary antibodies: rabbit polyclonal antibody against SIRT1 (diluted 1:100; Santa Cruz Biotechnology, Santa Cruz, CA, USA) or rabbit polyclonal antibody against iNOS (diluted 1:200; Santa Cruz Biotechnology, Santa Cruz, CA, USA) or simultaneously with goat antibody against SOD1 (diluted 1:100; Santa Cruz Biotechnology, Santa Cruz, CA, US) and rabbit polyclonal antibody against CAT (diluted $1: 150$; Santa Cruz Biotechnology, Santa Cruz, CA, US). Liver sections were then washed in tris-buffered saline (TBS) and, for immunohistochemical analysis of SIRT1 and iNOS, were incubated for 1 hour with specific biotinylated secondary antibody and after 1 hour with the avidin-biotin horseradish peroxidase complex (ABC-peroxidase kit Vector Labs, Burlingame, CA, USA), prepared according to the manufacturer's instructions. Finally, these liver sections were immersed in a solution of $0.05 \% 3^{\prime}-3^{\prime}$-diaminobenzidine tetrahydrochloride (DAB) and $0.03 \%$ hydrogen peroxide for 10 minutes, counterstained with haematoxylin, dehydrated, mounted, and observed with a light microscopy (Olympus, Hamburg, Germany) at a final magnification of 400x.

However, for the double immunofluorescence staining with SOD1 and CAT, the liver sections, after the incubation with primary antibodies, were labelled using anti-goat Alexa Fluor 546 and anti-rabbit Alexa Fluor 488 conjugated secondary antibodies (diluted 1:200; Invitrogen, UK). Finally, the sections were counterstained with $4^{\prime}$-6-diamidino-2phenylindole (DAPI), mounted, and observed with a confocal microscope (510 Meta Zeiss, Munich, Germany) at a final magnification of $400 \mathrm{x}$, as previously described by AgabitiRosei et al. [43].

Control reactions for both immunohistochemistry and immunofluorescence analysis were performed in absence of primary antibody and in the presence of isotype-matched IgGs.

Randomly chosen 20 liver fields for each experimental animal were analyzed and the immunostaining for each primary antibody was calculated using an image analyzer (Image Pro Premier 9.1, Media Cybernetics Inc., Rockville, USA). Two blinded investigators performed the histomorphometrical analysis and their evaluation was assumed to be correct if the values were not significantly different. If there was disagreement concerning the interpretation, the case was reconsidered to reach a unanimous agreement. The levels of immunostaining of each primary antibody evaluated were expressed as arbitrary units (AU).
2.5. Measurement of Serum Levels of Superoxide Dismutase1 and Glutathione. Serum samples were obtained collecting total blood in serum separator tubes and allowed samples to clot for 2 hours at room temperature. Then the samples were centrifuged for 20 minutes at $1000 \times \mathrm{g}$. The supernatants obtained were subjected to analyses of the levels of SOD1 and GSH through specific ELISA assay kits and following the respective manufacturer's instructions (LifeSpan BioSciences, Inc., Seattle, USA). In detail, the optical density values for both SOD1 and GSH were determined using a microplate reader set at $450 \mathrm{~nm}$. Furthermore, SOD1 ELISA assay presented intra-assay variations coefficient less than $4.7 \%$, interassay variations coefficient less than $9.5 \%$, and sensitivity less than $1.56 \mathrm{U} / \mathrm{mL}$, whereas the GSH ELISA assay had intra-assay variations coefficient less than $6.2 \%$, interassay variations coefficient less than $9.6 \%$, and sensitivity less than $1 \mathrm{ng} / \mathrm{mL}$.

2.6. Statistical Analysis. The data are expressed as mean \pm SD. All data were analyzed by one-way analyses of variance for repeated measures (ANOVA), corrected by Bonferroni, to compare the variability of a group with all other experimental groups. Probability values less than 0.05 were considered significant. All experiments were carried out in triplicate and data were collected and analyzed by Origin Pro 9.1 software (OriginLab Corporation, Northampton, MA, USA).

\section{Results}

3.1. Animal Treatment and Serum Biochemistry. The animal body weight measured before the start and at different time points of the treatment and also the liver weight measured at the end of the study did not show any significant difference among the experimental groups. Table 1 summarized the percentage of liver weight respect body weight.

As expected, ApoE $\mathrm{E}^{-/-} 6 \mathrm{w}$ and $15 \mathrm{w}$ had increased serum cholesterol and triglyceride levels compared to control mice. In particular, both serum cholesterol and triglyceride concentrations were higher in $\mathrm{ApoE}^{-/-} 15 \mathrm{w}$ with respect to $\mathrm{ApoE}^{-/-} 6 \mathrm{w}$. Interestingly, both serum parameters were significantly reduced after melatonin treatment of $\mathrm{ApoE}^{-/-}$mice (Table 1).

3.2. Haematoxylin-Eosin Staining. Haematoxylin-eosin staining showed that in untreated control C57BL/6 mice which were 6 weeks or 15 weeks old or in control C57BL/6 mice treated with $1 \%$ ethanol (vehicle of melatonin) or with melatonin presented a "normal" hepatic parenchyma without LD. These observations underline that all the above reported experimental groups are similar, so they are considered a single group defined generically as "control" in the following morphological, immunohistochemical/immunofluorescence, and histomorphometrical analysis.

In both untreated $\mathrm{ApoE}^{-/-} 6 \mathrm{w}$ and $15 \mathrm{w}$ groups, we observed significant LD. In fact, both untreated groups presented hepatocyte ballooning, nuclear polyploidy, and granular cytoplasm with prevalently small lipid droplets deposition, characteristic of microvesicular steatosis, together with 
TABLE 1: Liver/body weight ratio and serum parameters.

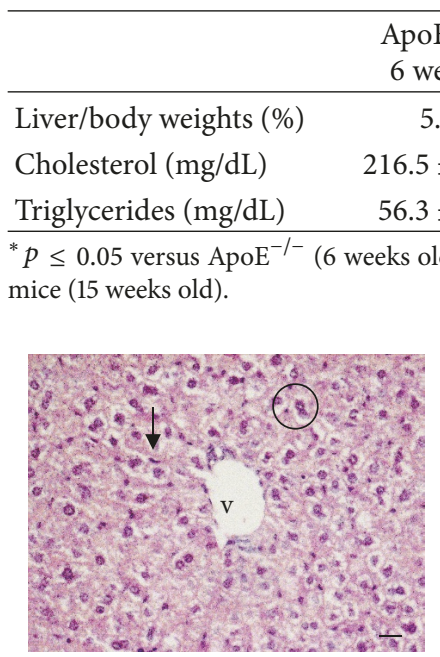

(a)

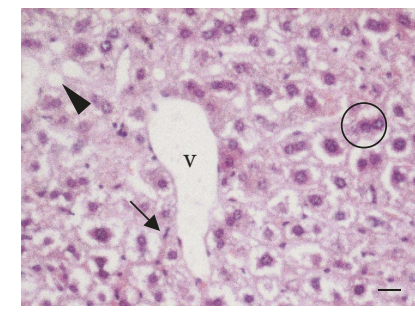

(b)

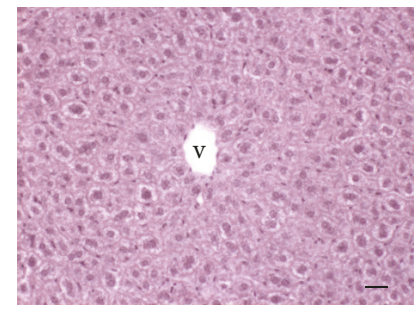

(c)

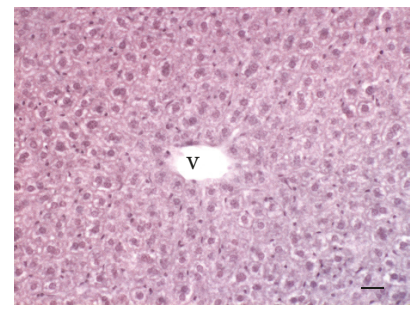

(d)

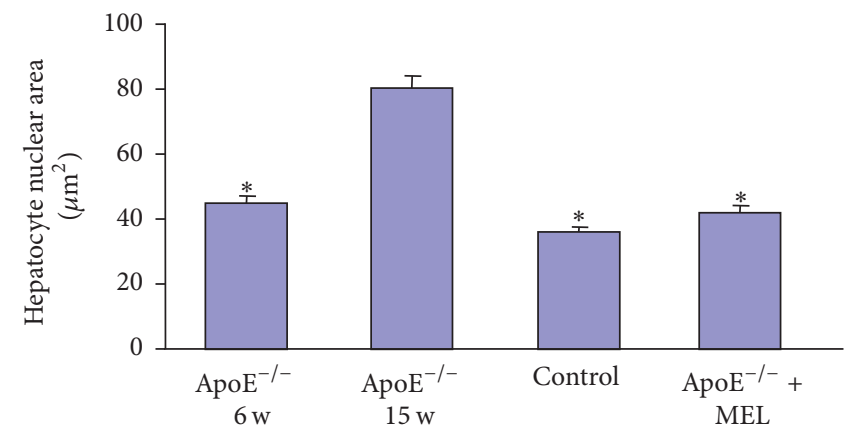

(e)

Figure 1: Photomicrographs of liver haematoxylin-eosin staining of untreated $\mathrm{ApoE}^{-/-}$of $6 \mathrm{w}$ (a), untreated ApoE $\mathrm{E}^{-/-}$of $15 \mathrm{w}$ (b), control (c), and $\mathrm{ApoE}^{-1-}+$ MEL (d). Bar: $20 \mu \mathrm{m}$. v: central vein, the arrowhead indicates lipid droplet deposition, the arrow identifies the inflammatory cells infiltration, and the circle indicates hepatocyte nuclear polyploidy. The graph (e) summarized the morphometrical analysis of hepatocyte nuclear area. * $p \leq 0.05$ versus $\mathrm{ApoE}^{-/-}$of $15 \mathrm{w}$.

inflammatory cells infiltration. The reported hepatic parenchymal alterations were weak/moderate in $\mathrm{ApoE}^{-/-} 6 \mathrm{w}$ (Figure 1(a)) and moderate/strong in $\mathrm{ApoE}^{-/-} 15 \mathrm{w}$ (Figure 1(b)), with respect to liver of control mice that showed "normal" hepatic parenchyma with radially arranged rays of hepatocytes, regularly directed from the central vein of each lobule towards its periphery (Figure 1(c)). Liver lipid droplets deposition, hepatocyte ballooning, nuclear polyploidy, and inflammatory infiltration were significantly decreased in liver of $\mathrm{ApoE}^{-/-}+\mathrm{MEL}$, exhibiting an almost "normal" hepatic cytoarchitecture (Figure 1(d)).

Furthermore, untreated $\mathrm{ApoE}^{-/-} 15 \mathrm{w}$ showed a significant increased hepatocyte nuclear area with respect to both $\mathrm{ApoE}^{-/-} 6 \mathrm{w}$ and control groups. Remarkably, liver of $\mathrm{ApoE}^{-/-}+$MEL exhibited a significant hepatocyte nuclear area reduction, reaching value comparable to liver of control mice (Figure 1(e)).

3.3. Picrosirius Red Staining. Picrosirius red staining showed a mild parenchymal hepatic perisinusoidal fibrosis in untreated $\mathrm{ApoE}^{-/-} 6 \mathrm{w}$ (Figure 2(a)) and a moderate/strong perisinusoidal collagen deposition in liver of untreated $\mathrm{ApoE}^{-/-} 15 \mathrm{w}$ (Figure 2(b)) with respect to liver of control mice that showed very weak parenchymal fibrosis (Figure 2(c)). The liver of $\mathrm{ApoE}^{-/-}+$MEL exhibited a significant fibrosis reduction (very weak collagen deposition) (Figure $2(d))$.

3.4. Immunohistochemical Analysis of Sirtuin1. Immunohistochemical analysis of SIRT1 showed a very weak/weak expression at parenchymal liver level of both untreated $\mathrm{ApoE}^{-/-} 6 \mathrm{w}$ (Figure 3(a)) and untreated $\mathrm{ApoE}^{-/-} 15 \mathrm{w}$ (Figure 3(b)), relative to liver of control mice that exhibited diffuse and strong expression at both hepatocytes cytoplasm and nuclear level (Figure 3(c)). The liver of $\mathrm{ApoE}^{-/-}+\mathrm{MEL}$ exhibited a significant increase in SIRT1 expression at both cytoplasm and nuclear levels (Figure 3(d)), reaching level almost comparable to liver of control mice.

These observations were confirmed also by liver SIRT1 histomorphometrical analysis (Figure 3(e)). 


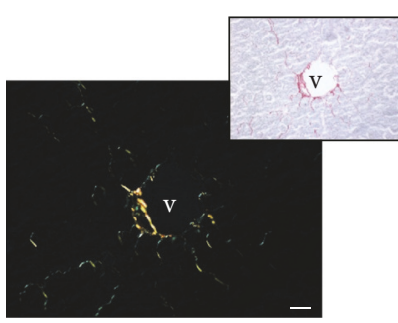

(a)

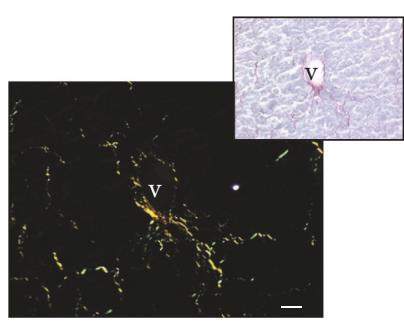

(b)

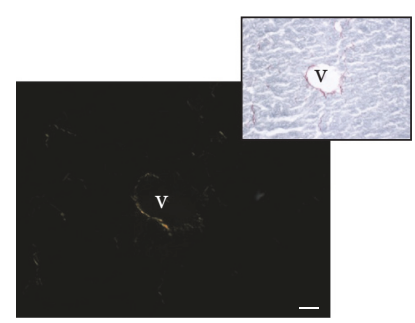

(c)

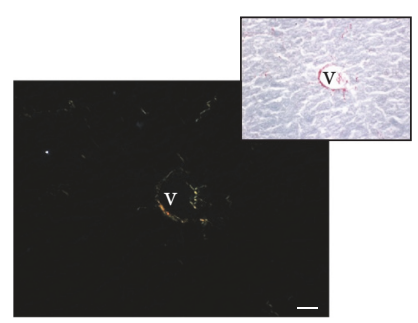

(d)

Figure 2: Photomicrographs of liver Picrosirius red staining of untreated $\mathrm{ApoE}^{-/-}$of $6 \mathrm{w}$ (a), untreated ApoE ${ }^{-/-}$of $15 \mathrm{w}$ (b), control (c), and $\mathrm{ApoE}^{-/-}+\mathrm{MEL}(\mathrm{d})$ under polarized light (a-d) or without polarized light (inserts). Bar: $20 \mu \mathrm{m}$. v: central vein.

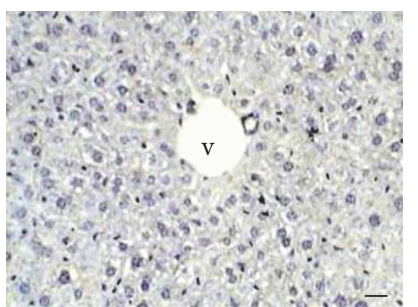

(a)

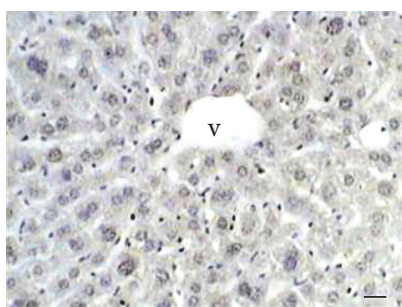

(b)

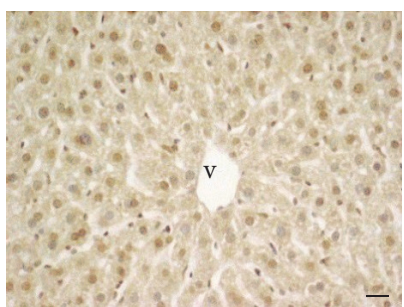

(c)

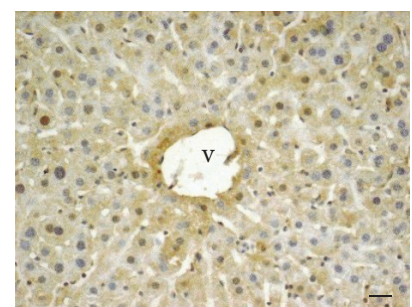

(d)

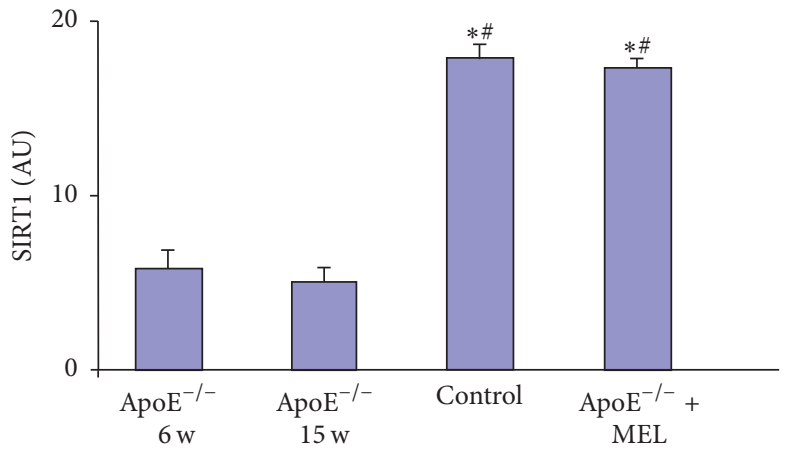

(e)

FIgURE 3: Photomicrographs of liver SIRT1 immunohistochemical analysis of ApoE $\mathrm{E}^{-/-}$of $6 \mathrm{w}(\mathrm{a}), \mathrm{ApoE}^{-/-}$of $15 \mathrm{w}$ (b), control (c), and ApoE ${ }^{-/-}$ + MEL (d). Bar: $20 \mu \mathrm{m}$. v: central vein. The graph (e) summarizes the SIRT1 histomorphometrical analysis. ${ }^{*} p \leq 0.05$ versus ApoE ${ }^{-/-}$of $6 \mathrm{w}$ and ${ }^{\#} p \leq 0.05$ versus $A \mathrm{poE}^{-/-}$of $15 \mathrm{w}$.

3.5. Immunohistochemical Analysis of Oxidative Stress Marker (iNOS) and Double Immunofluorescence Analysis of Antioxidants (SOD1 and CAT). Both untreated $A_{\text {poE }}{ }^{-/-} 6 \mathrm{w}$ and $15 \mathrm{w}$ groups showed a significant iNOS expression at parenchymal liver level; in particular, liver of untreated $\mathrm{ApoE}^{-/-} 6 \mathrm{w}$ had a moderate iNOS expression (Figure 4(a)) and liver of untreated $\mathrm{ApoE}^{-/-} 15 \mathrm{w}$ exhibited a strong expression (Figure 4(b)), relative to liver of control mice that showed very weak/weak iNOS expression (Figure 4(c)). Remarkably, liver of $\mathrm{ApoE}^{-/-}+$MEL showed a significant reduction of iNOS expression (Figure 4(d)), similar to liver of control mice. The hepatic iNOS expression was localized prevalently at hepatocyte cytoplasm throughout the hepatic lobule.

The double immunofluorescence evaluation of endogenous antioxidants SOD1 (identified in green staining) and CAT (identified in red staining) showed that parenchymal liver of untreated $\mathrm{ApoE}^{-/-} 6 \mathrm{w}$ had a mild hepatic expression of SOD1 and a very weak/weak CAT expression (Figure 4(e)). Instead, liver of untreated $\mathrm{ApoE}^{-/-} 15 \mathrm{w}$ showed very weak/weak expression of both antioxidants (Figure 4(f)) with respect to liver of control mice that had diffuse and strong parenchymal expression of both SOD1 and CAT (Figure $4(\mathrm{~g})$ ). Interestingly, liver of $\mathrm{ApoE}^{-/-}+\mathrm{MEL}$ showed a significant increase of both antioxidants (Figure 4(h)), reaching values comparable to liver of control mice. Both antioxidants were expressed at hepatocyte cytoplasm level and no nuclear positivity was observed.

All the above reported observations were confirmed also by the histomorphometrical hepatic analysis of iNOS (Figure 4(i)), SOD1 (Figure 4(j)), and CAT (Figure 4(k)).

3.6. Serum Levels of Superoxide Dismutase1 and Glutathione. Next to histomorphometrical analyses of SOD1 and CAT, we evaluated also the serum levels of the antioxidant enzymes 


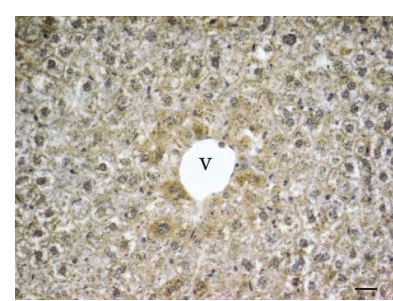

(a)

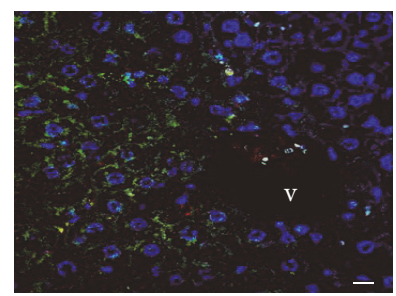

(e)

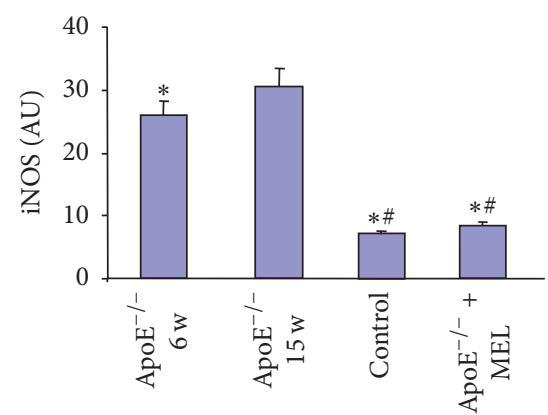

(i)

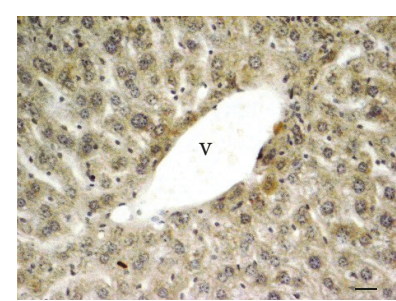

(b)

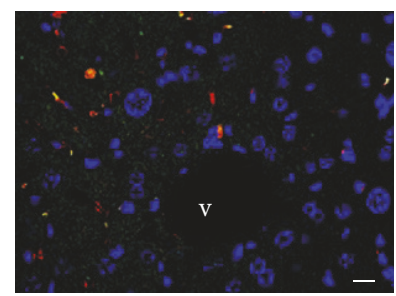

(f)

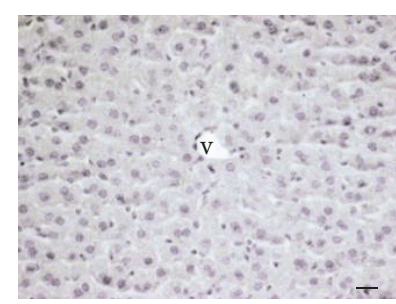

(c)

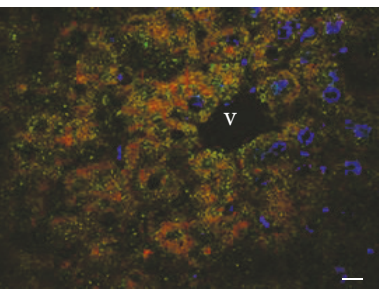

(g)

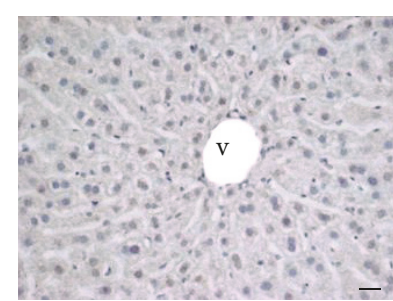

(d)

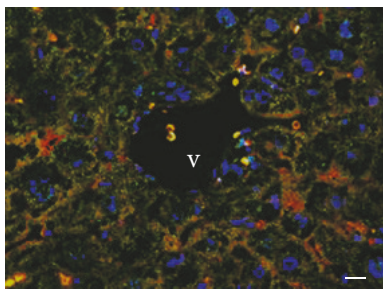

(h)

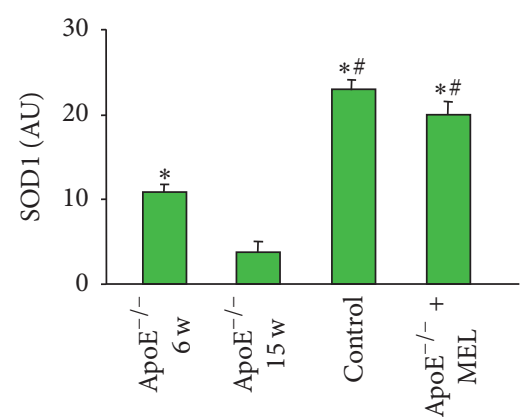

(j)

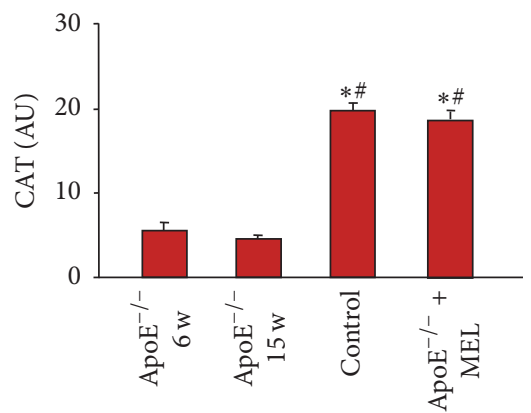

(k)

FIGURE 4: Photomicrographs of liver immunohistochemical analysis of iNOS (a-d) and of liver double immunofluorescence analysis of SOD1 (green staining) and CAT (red staining) (e-h) of $\mathrm{ApoE}^{-/-}$of $6 \mathrm{w}(\mathrm{a}, \mathrm{e}), \mathrm{ApoE}^{-/-}$of $15 \mathrm{w}$ (b, f), control (c, g), and ApoE $\mathrm{E}^{-/-}+\mathrm{MEL}(\mathrm{d}, \mathrm{h}) . \mathrm{Bar}$ : $20 \mu \mathrm{m}$. v: central vein. The graphs summarized the iNOS (i), SOD1 (j), and CAT (k) histomorphometrical analysis. ${ }^{*} p \leq 0.05 \mathrm{versus} \mathrm{ApoE}^{-/-}$ of $6 \mathrm{w}$ and ${ }^{\#} p \leq 0.05$ versus $\mathrm{ApoE}^{-/-}$of $15 \mathrm{w}$.

SOD1 and GSH. ApoE ${ }^{-/-} 15 \mathrm{w}$ showed a significant reduction of both SOD1 and GSH levels with respect to control mice that showed strong/moderate serum levels. Remarkably, melatonin treatment of $\mathrm{ApoE}^{-/-}$mice showed a significant increase of both serum antioxidants (strong levels). Figure 5(a) summarized SOD1 serum levels, whereas GSH serum levels are represented in Figure 5(b).

\section{Discussion}

The LD observed in an $\mathrm{ApoE}^{-/-}$hypercholesterolemic mouse model were significantly reduced by oral supplementation of melatonin.

We observed a severe decrease of SIRT1 expression in liver of $\mathrm{ApoE}^{-/-}$mice, more evident at 15 weeks of life. Growing evidences from both human and experimental studies revealed that impaired SIRT1 signaling is associated with alcoholic liver disease and genetic or pharmacological stimulations of SIRT1 protect against steatosis and/or steatohepatitis $[44,45]$. Furthermore, liver-specific SIRT1 deficiency is related to hepatic glucose overproduction, chronic hyperglycemia, and increased OS [12, 22].
To highlight the importance of hepatic SIRT1 expression and its involvement in OS, we evaluated the expression of iNOS, a proinflammatory protein commonly related to OS status, and of endogenous antioxidants (SOD1, CAT, and GSH). Our results showed that iNOS is strongly expressed in liver of $\mathrm{ApoE}^{-/-}$mice and this increase plays, in turn, a critical role in the development of liver inflammation and OS $[46,47]$. We observed also a significant low expression of antioxidant enzymes in $\mathrm{ApoE}^{-/-} 15 \mathrm{w}$ both in hepatic parenchyma and at serum level. It is known that, under physiological conditions, these enzymes can efficiently counteract oxidative damage induced by free radicals, while in pathological conditions there are no enough endogenous antioxidants to counteract the excessive free radicals [48].

Remarkably, our results exhibited that the oral supplementation of melatonin restored SIRT1 hepatic expression that, in turn, had an antagonistic effect to LD and OS in $\mathrm{ApoE}^{-/-}$mice. These findings confirmed the literature data, reporting that melatonin had important antioxidant effects and also a strong tendency to attenuate hepatic steatosis [49]. In our opinion, the protective effects of melatonin against LD related to HC may be attributed not only to its direct 


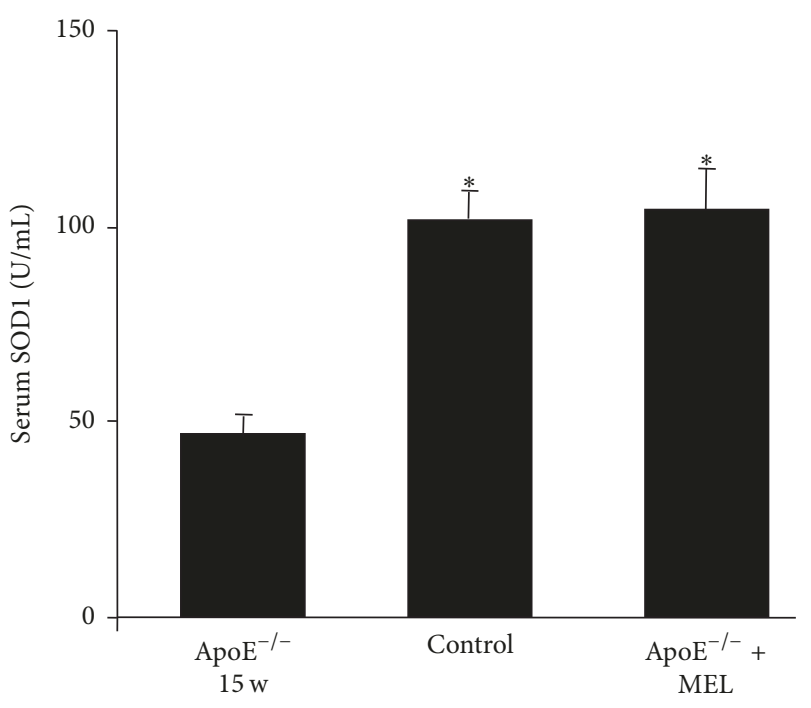

(a)

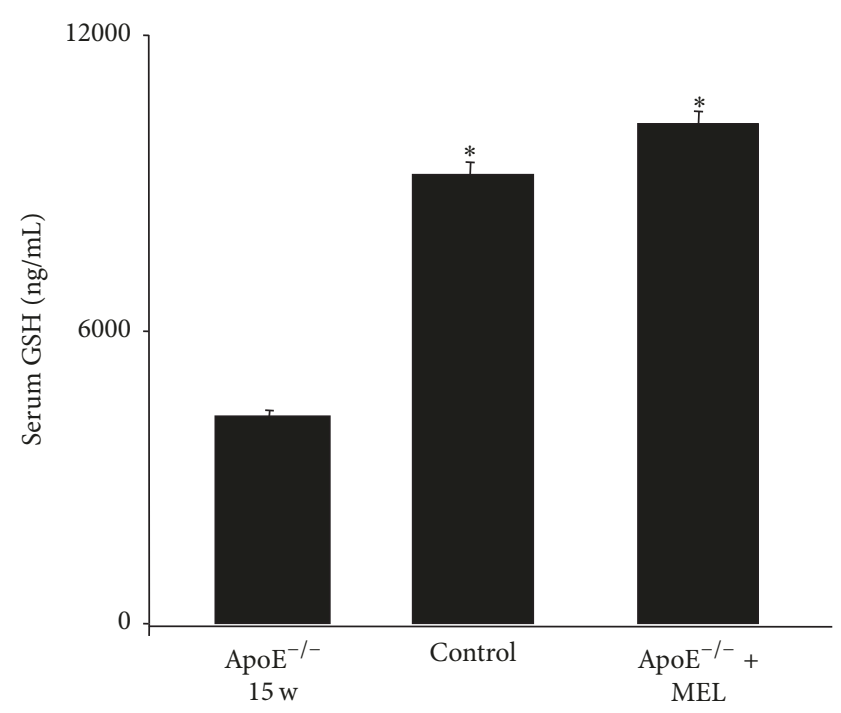

(b)

FIGURE 5: Serum superoxide dismutasel (SOD1) (a) and glutathione (GSH) (b) levels. ${ }^{*} p \leq 0.05$ versus ApoE ${ }^{-/-}$of 15 w.

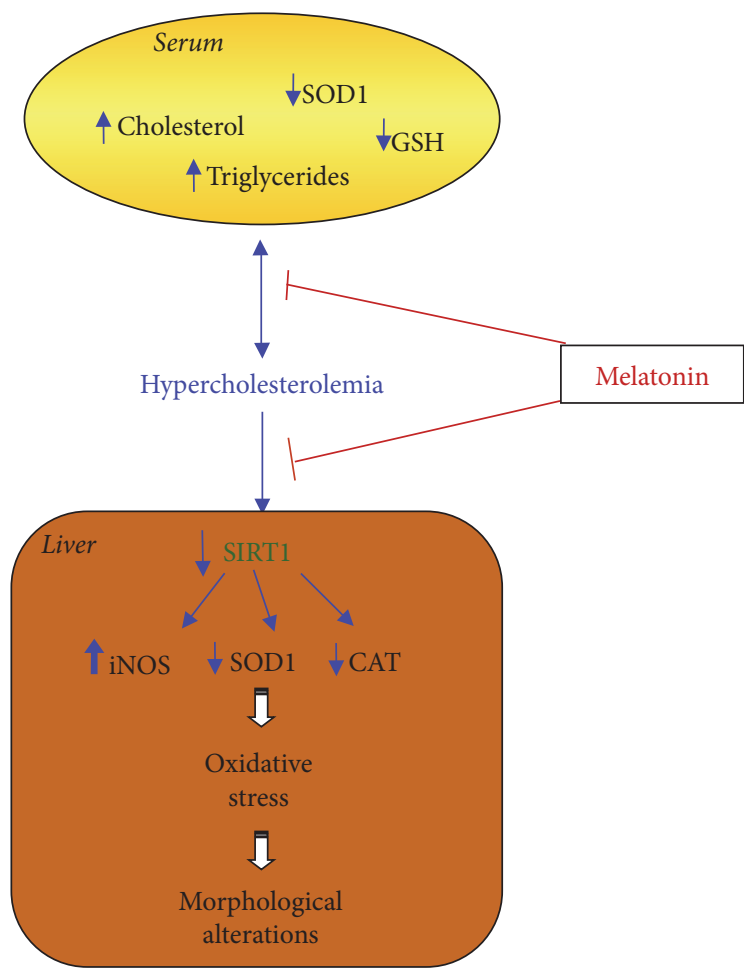

FIGURE 6: Schematic representation of melatonin effects against hypercholesterolemic hepatic alterations. It is important to underline that this indoleamine, together with its antioxidant properties, is able to reduce liver morphological alterations also blocking the decrease of sirtuinl that in turn inhibits oxidative stress. The red symbols indicate the effect of melatonin in blocking the pathways induced by hypercholesterolemia that are indicated by the blue arrows. CAT: catalase; GSH: glutathione; iNOS: inducible nitric oxide synthase; SIRT1: sirtuin1; SOD1: superoxide dismutase1.

and/or indirect antioxidant properties, but probably also through its action mediated by SIRT1 that is involved in the inhibition of iNOS and preservation of the endogenous antioxidant system. Moreover, our results are in agreement with Lee et al. [50] who showed that treatment of a $\beta$-cell line (RIN) with cytokines induced cell damage correlated with the increased iNOS expression. However, SIRT1 overexpression completely prevented cytotoxicity and the increase of iNOS by the inhibition of the nuclear factor- $\mathrm{kB}$ signaling. Tanno et al. [51] also showed that SIRT1 participate in the modulation of the endogenous antioxidants.

Other experimental studies showed that melatonin alone or administered in combination with pioglitazone or pentoxifylline reduced the insulin resistance index and total cholesterol and triglycerides and modulated GSH level during NAFLD [52].

\section{Conclusion}

Our results suggest that oral supplementation of melatonin inhibits LD induced by HC and that not only could this effect be due to its known antioxidant properties, but also it may be mediated by a SIRT1-dependent mechanism that, in turn, blocks serum cholesterol and triglycerides increase and hepatic iNOS expression and restores endogenous antioxidants (Figure 6). Further studies are needed for addressing the involvement of SIRT1 in HC and the mechanism(s) by which melatonin counteracts LD.

\section{Conflicts of Interest}

The authors declare that there are no conflicts of interest regarding the publication of this manuscript.

\section{Authors' Contributions}

Francesca Bonomini and Gaia Favero contributed equally to the present work. 


\section{Acknowledgments}

Thanks are due to Lorena Giugno and Stefania Castrezzati for their technical support. This study was supported by the University of Brescia, Italy, Grant (ex 60\%).

\section{References}

[1] C. M. Balarini, M. Z. T. Oliveira, T. M. C. Pereira et al., "Hypercholesterolemia promotes early renal dysfunction in apolipoprotein E-deficient mice," Lipids in Health and Disease, vol. 10, article no. 220, 2011.

[2] M. Gaggini, M. Morelli, E. Buzzigoli, R. A. DeFronzo, E. Bugianesi, and A. Gastaldelli, "Non-alcoholic fatty liver disease (NAFLD) and its connection with insulin resistance, dyslipidemia, atherosclerosis and coronary heart disease," Nutrients, vol. 5, no. 5, pp. 1544-1560, 2013.

[3] E. Ahishali, K. Demir, B. Ahishali et al., "Electron microscopic findings in non-alcoholic fatty liver disease: Is there a difference between hepatosteatosis and steatohepatitis?" Journal of Gastroenterology and Hepatology, vol. 25, no. 3, pp. 619-626, 2010.

[4] C. M. Depner, M. Torres-Gonzalez, S. Tripathy, G. Milne, and D. B. Jump, "Menhaden oil decreases high-fat diet-induced markers of hepatic damage, steatosis, inflammation, and fibrosis in obese $\mathrm{Ldll}^{-/-}$mice," Journal of Nutrition, vol. 142, no. 8, pp. 1495-1503, 2012.

[5] K. A. Lytle, C. P. Wong, and D. B. Jump, "Docosahexaenoic acid blocks progression of western diet-induced nonalcoholic steatohepatitis in obese $\mathrm{Ldlr}^{-/-}$mice," PLoS ONE, vol. 12, no. 4, Article ID e0173376, 2017.

[6] M. Pérez-Carreras, P. Del Hoyo, M. A. Martín et al., "Defective hepatic mitochondrial respiratory chain in patients with nonalcoholic steatohepatitis," Hepatology, vol. 38, no. 4, pp. 999-1007, 2003.

[7] S. Seki, T. Kitada, T. Yamada, H. Sakaguchi, K. Nakatani, and K. Wakasa, "In situ detection of lipid peroxidation and oxidative DNA damage in non-alcoholic fatty liver diseases," Journal of Hepatology, vol. 37, no. 1, pp. 56-62, 2002.

[8] H. L. Reeves, A. D. Burt, S. Wood, and C. P. Day, "Hepatic stellate cell activation occurs in the absence of hepatitis in alcoholic liver disease and correlates with the severity of steatosis," Journal of Hepatology, vol. 25, no. 5, pp. 677-683, 1996.

[9] H. Wobser, C. Dorn, T. S. Weiss et al., "Lipid accumulation in hepatocytes induces fibrogenic activation of hepatic stellate cells," Cell Research, vol. 19, no. 8, pp. 996-1005, 2009.

[10] M. Esrefoglu, A. Cetin, E. Taslidere et al., "Therapeutic effects of melatonin and quercetin in improvement of hepatic steatosis in rats through supression of oxidative damage," Bratislava Medical Journal, vol. 118, no. 6, pp. 347-354, 2017.

[11] F. M. Mauren, Yanti, and B. W. Lay, "Efficacy of oral curcuminoid fraction from curcuma xanthorrhiza and curcuminoid cider in high-cholesterol fed rats," Pharmacognosy Research, vol. 8, no. 3, pp. 153-159, 2016.

[12] K. Sodhi, N. Puri, G. Favero et al., "Fructose mediated nonalcoholic fatty liver is attenuated by HO-1-SIRT1 module in murine hepatocytes and mice fed a high fructose diet," PLoS ONE, vol. 10, no. 6, Article ID e0128648, 2015.

[13] F. Bonomini, L. F. Rodella, M. Moghadasian, C. Lonati, and R. Rezzani, "Apolipoprotein e deficiency and a mouse model of accelerated liver aging," Biogerontology, vol. 14, no. 2, pp. 209220, 2013.
[14] C. Csonka, T. Baranyai, L. Tiszlavicz et al., "Isolated hypercholesterolemia leads to steatosis in the liver without affecting the pancreas," Lipids in Health and Disease, vol. 16, no. 1, article no. 144, 2017.

[15] S. E. Choi, S. Kwon, S. Seok et al., "Obesity-linked phosphorylation of SIRT1 by casein kinase 2 inhibits its nuclear localization and promotes fatty liver," Molecular and Cellular Biology, vol. 37, no. 15, Article ID e00006-17, 2017.

[16] F. Nassir and J. A. Ibdah, "Sirtuins and nonalcoholic fatty liver disease," World Journal of Gastroenterology, vol. 22, no. 46, pp. 10084-10092, 2016.

[17] Y. Zhang, C. Geng, X. Liu et al., "Celastrol ameliorates liver metabolic damage caused by a high-fat diet through Sirtl," Molecular Metabolism, vol. 6, no. 1, pp. 138-147, 2017.

[18] N. Puri, K. Sodhi, M. Haarstad et al., "Heme induced oxidative stress attenuates sirtuinl and enhances adipogenesis in mesenchymal stem cells and mouse pre-adipocytes," Journal of Cellular Biochemistry, vol. 113, no. 6, pp. 1926-1935, 2012.

[19] B. Ponugoti, D. H. Kim, Z. Xiao et al., "SIRT1 deacetylates and inhibits SREBP-1C activity in regulation of hepatic lipid metabolism," The Journal of Biological Chemistry, vol. 285, no. 44, pp. 33959-33970, 2010.

[20] Y. Yamazaki, I. Usui, Y. Kanatani et al., "Treatment with SRT1720, a SIRT1 activator, ameliorates fatty liver with reduced expression of lipogenic enzymes in MSG mice," American Journal of Physiology-Endocrinology and Metabolism, vol. 297, no. 5, pp. E1179-E1186, 2009.

[21] K. E. Kim, H. Kim, R. W. Heo et al., "Myeloid-specific SIRT1 deletion aggravates hepatic inflammation and steatosis in highfat diet-fed mice," Korean Journal of Physiology \& Pharmacology, vol. 19, no. 5, pp. 451-460, 2015.

[22] S. Chung, H. Yao, S. Caito, J.-W. Hwang, G. Arunachalam, and I. Rahman, "Regulation of SIRT1 in cellular functions: role of polyphenols," Archives of Biochemistry and Biophysics, vol. 501, no. 1, pp. 79-90, 2010.

[23] T. Tobita, J. Guzman-Lepe, K. Takeishi et al., "SIRT1 disruption in human fetal hepatocytes leads to increased accumulation of glucose and lipids," PLoS ONE, vol. 11, no. 2, Article ID e0149344, 2016.

[24] A. Purushotham, T. T. Schug, Q. Xu, S. Surapureddi, X. Guo, and X. Li, "Hepatocyte-specific deletion of SIRT1 alters fatty acid metabolism and results in hepatic steatosis and inflammation," Cell Metabolism, vol. 9, no. 4, pp. 327-338, 2009.

[25] V. K. Awasthi, F. Mahdi, R. Chander et al., "Hypolipidemic Activity of Cassia tora Seeds in Hyperlipidemic Rats," Indian Journal of Clinical Biochemistry, vol. 30, no. 1, pp. 78-83, 2015.

[26] S. Ampawong, D. Isarangkul, and P. Aramwit, "Sericin improves heart and liver mitochondrial architecture in hypercholesterolaemic rats and maintains pancreatic and adrenal cell biosynthesis," Experimental Cell Research, vol. 358, no. 2, pp. 301-314, 2017.

[27] J. Barrenetxe, P. Delagrange, and J. A. Martínez, "Physiological and metabolic functions of melatonin," Journal of Physiology and Biochemistry, vol. 60, no. 1, pp. 61-72, 2004.

[28] R. Hardeland, D. P. Cardinali, V. Srinivasan, D. W. Spence, G. M. Brown, and S. R. Pandi-Perumal, "Melatonin-a pleiotropic, orchestrating regulator molecule," Progress in Neurobiology, vol. 93, no. 3, pp. 350-384, 2011.

[29] R. J. Reiter, J. C. Mayo, D.-X. Tan, R. M. Sainz, M. AlatorreJimenez, and L. Qin, "Melatonin as an antioxidant: under 
promises but over delivers," Journal of Pineal Research, pp. 253278, 2016.

[30] B. de Luxán-Delgado, Y. Potes, A. Rubio-González et al., "Melatonin reduces endoplasmic reticulum stress and autophagy in liver of leptin-deficient mice," Journal of Pineal Research, vol. 61, pp. 108-123, 2016.

[31] A. Stacchiotti, G. Favero, A. Lavazza et al., "Hepatic macrosteatosis is partially converted to microsteatosis by melatonin supplementation in ob/ob mice non-alcoholic fatty liver disease," PLoS ONE, vol. 11, no. 1, Article ID e0148115, 2016.

[32] L. F. Rodella, G. Favero, C. Rossini et al., "Aging and vascular dysfunction: Beneficial melatonin effects," $A G E$, vol. 35 , no. 1 , pp. 103-115, 2013.

[33] M. Tajes, J. Gutierrez-Cuesta, D. Ortuño-Sahagun, A. Camins, and M. Pallàs, "Anti-aging properties of melatonin in an in vitro murine senescence model: Involvement of the sirtuin 1 pathway," Journal of Pineal Research, vol. 47, no. 3, pp. 228-237, 2009.

[34] F. Bonomini, S. Taurone, P. Parnigotto et al., "Role of parnaparin in atherosclerosis," International Journal of Clinical and Experimental Pathology, vol. 97, no. 6, pp. 457-464, 2016.

[35] Y. Ning, L. Xu, S. Ren, W. M. Pandak, S. Chen, and L. Yin, "StAR overexpression decreases serum and tissue lipids in apolipoprotein E-deficient mice," Lipids, vol. 44, no. 6, pp. 511519, 2009.

[36] N. Ferré, M. Martínez-Clemente, M. López-Parra et al., "Increased susceptibility to exacerbated liver injury in hypercholesterolemic ApoE-deficient mice: Potential involvement of oxysterols," American Journal of Physiology-Gastrointestinal and Liver Physiology, vol. 296, no. 3, pp. G553-G562, 2009.

[37] C. Parolini, B. Bjorndal, M. Busnelli et al., "Effect of dietary components from antarctic krill on atherosclerosis in apoEdeficient mice," Molecular Nutrition \& Food Research, vol. 61, no. 12, p. 1700098, 2017.

[38] R. Rezzani, G. Favero, A. Stacchiotti, and L. F. Rodella, "Endothelial and vascular smooth muscle cell dysfunction mediated by cyclophylin A and the atheroprotective effects of melatonin," Life Sciences, vol. 92, no. 17-19, pp. 875-882, 2013.

[39] R. Milcheva, P. Janega, P. Celec, R. Russev, and P. Babál, "Alcohol based fixatives provide excellent tissue morphology, protein immunoreactivity and RNA integrity in paraffin embedded tissue specimens," Acta Histochemica, vol. 115, no. 3, pp. 279289, 2013.

[40] A. Mocali, L. Giovannelli, P. Dolara, and F. Paoletti, “The comet assay approach to senescent human diploid fibroblasts identifies different phenotypes and clarifies relationships among nuclear size, DNA content, and DNA damage," The Journals of Gerontology. Series A, Biological Sciences and Medical Sciences, vol. 60, no. 6, pp. 695-701, 2005.

[41] J. A. Lidbury, A. Rodrigues Hoffmann, R. Ivanek et al., "Interobserver Agreement Using Histological Scoring of the Canine Liver," Journal of Veterinary Internal Medicine, vol. 31, no. 3, pp. 778-783, 2017.

[42] D. Rizzoni, E. Porteri, C. De Ciuceis et al., "Effect of treatment with candesartan or enalapril on subcutaneous small artery structure in hypertensive patients with noninsulin-dependent diabetes mellitus," Hypertension, vol. 45, no. 4, pp. 659-665, 2005.

[43] C. Agabiti-Rosei, G. Favero, C. De Ciuceis et al., "Effect of longterm treatment with melatonin on vascular markers of oxidative stress/inflammation and on the anticontractile activity of perivascular fat in aging mice," Hypertension Research, vol. 40, no. 1, pp. 41-50, 2017.

[44] S. Timmers, E. Konings, L. Bilet et al., "Calorie restrictionlike effects of 30 days of resveratrol supplementation on energy metabolism and metabolic profile in obese humans," Cell Metabolism, vol. 14, no. 5, pp. 612-622, 2011.

[45] H. Yin, M. Hu, X. Liang et al., "Deletion of SIRT1 from hepatocytes in mice disrupts lipin-1 signaling and aggravates alcoholic fatty liver," Gastroenterology, vol. 146, no. 3, pp. 801-811, 2014.

[46] E. Talavera-Urquijo, S. Rodríguez-Navarro, M. Beisani et al., "Morphofunctional Changes After Sleeve Gastrectomy and Very Low Calorie Diet in an Animal Model of Non-Alcoholic Fatty Liver Disease," Obesity Surgery, pp. 1-10, 2017.

[47] X. Wang, M. Hausding, S. Weng et al., "Gliptins suppress inflammatory macrophage activation to mitigate inflammation, fibrosis, oxidative stress, and vascular dysfunction in models of nonalcoholic steatohepatitis and liver Fibrosis," Antioxidants \& Redox Signaling, vol. 28, no. 2, pp. 87-109, 2018.

[48] S. Y. Zhu, Y. Dong, J. Tu, Y. Zhou, X. H. Zhou, and B. Xu, "Silybum marianum oil attenuates oxidative stress and ameliorates mitochondrial dysfunction in mice treated with D-galactose," Pharmacognosy Magazine, vol. 10, no. 37, pp. S92-S99, 2014.

[49] G. Hatzis, P. Ziakas, N. Kavantzas et al., "Melatonin attenuates high fat diet-induced fatty liver disease in rats," World Journal of Hepatology, vol. 5, no. 4, pp. 160-169, 2013.

[50] J.-H. Lee, M.-Y. Song, E.-K. Song et al., "Overexpression of SIRT1 protects pancreatic $\beta$-cells against cytokine toxicity by suppressing the nuclear factor- $\kappa \mathrm{B}$ signaling pathway," Diabetes, vol. 58, no. 2, pp. 344-351, 2009.

[51] M. Tanno, A. Kuno, Y. Horio, and T. Miura, "Emerging beneficial roles of sirtuins in heart failure," Basic Research in Cardiology, vol. 107, no. 4, article no. 273, 2012.

[52] S. Zaitone, N. Hassan, N. El-Orabi, and E.-S. El-Awady, "Pentoxifylline and melatonin in combination with pioglitazone ameliorate experimental non-alcoholic fatty liver disease," European Journal of Pharmacology, vol. 662, no. 1-3, pp. 70-77, 2011. 


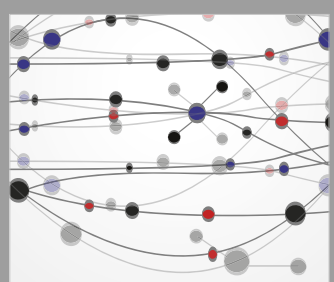

The Scientific World Journal
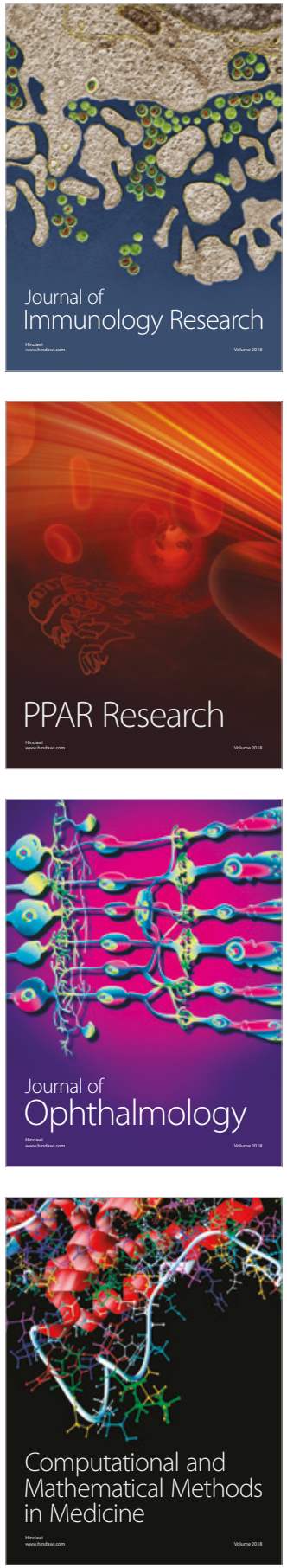

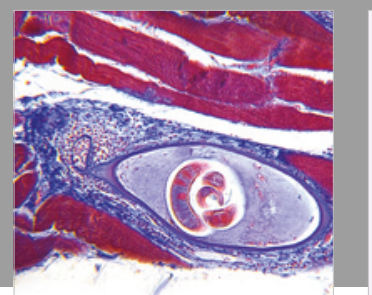

Gastroenterology Research and Practice

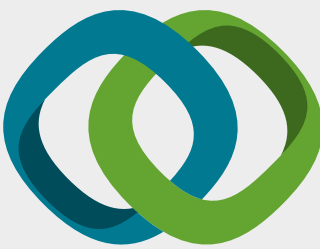

\section{Hindawi}

Submit your manuscripts at

www.hindawi.com
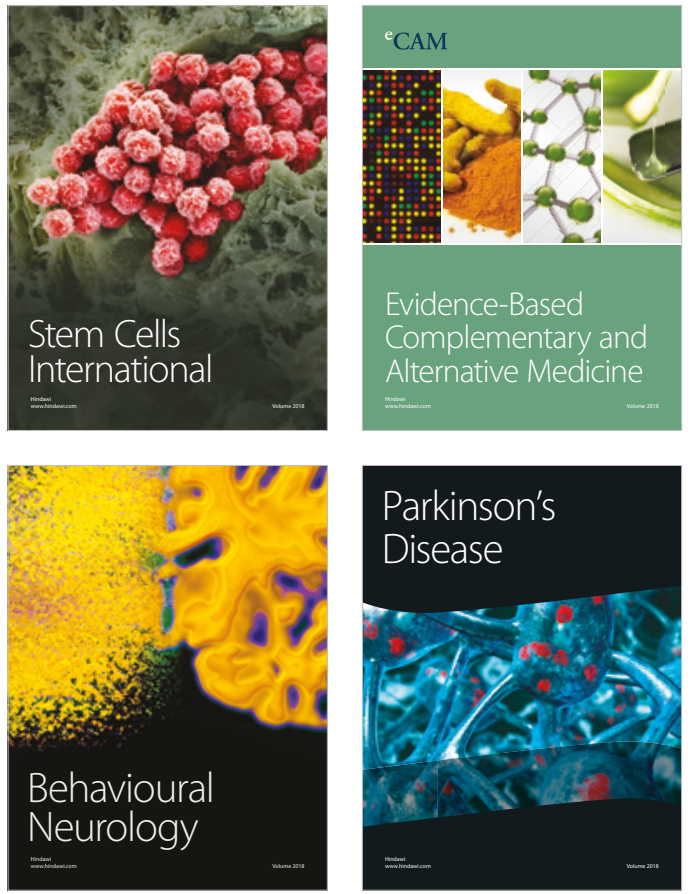

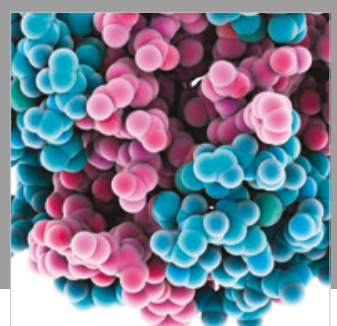

ournal of

Diabetes Research

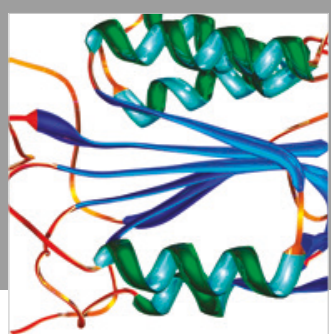

Disease Markers
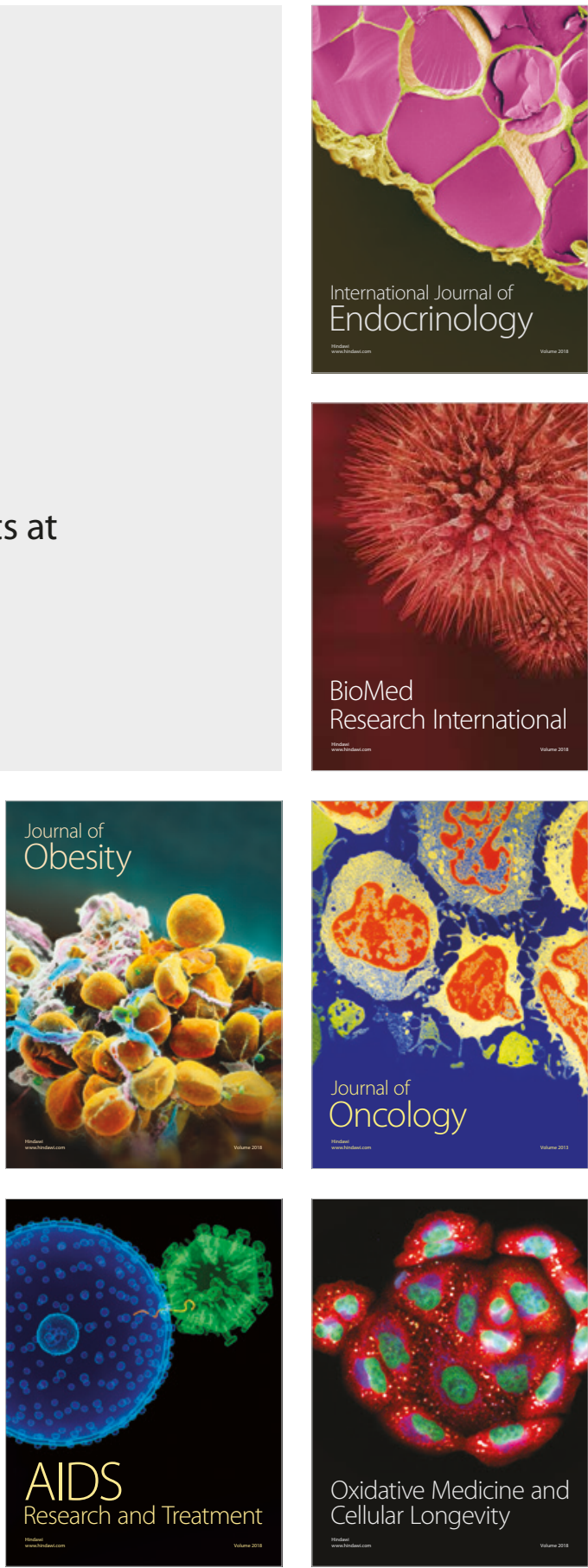